\section{$\underset{\substack{\text { hommes } \\ \text { \& migrations }}}{ }$}

\section{Hommes \& migrations}

Revue française de référence sur les dynamiques

migratoires

\section{6-1287 | 2010}

Les migrations subsahariennes

\title{
Papa Wemba
}

Retour d'une grande voix

\section{François Bensignor}

\section{OpenEdition \\ 12 Journals}

Édition électronique

URL : http://journals.openedition.org/hommesmigrations/1671

DOI : 10.4000/hommesmigrations. 1671

ISSN : 2262-3353

\section{Éditeur}

Musée national de l'histoire de l'immigration

\section{Édition imprimée}

Date de publication : 1 juillet 2010

Pagination : 296-301

ISSN : 1142-852X

\section{Référence électronique}

François Bensignor, «Papa Wemba », Hommes \& migrations [En ligne], 1286-1287 | 2010, mis en ligne le 29 mai 2013, consulté le 22 septembre 2020. URL : http://journals.openedition.org/

hommesmigrations/1671; DOI : https://doi.org/10.4000/hommesmigrations.1671

Ce document a été généré automatiquement le 22 septembre 2020.

Tous droits réservés 


\title{
Papa Wemba
}

\author{
Retour d'une grande voix
}

\author{
François Bensignor
}

1 Pour de nombreux amateurs de musiques du monde, le nom de Papa Wemba évoque ce grain de voix inimitable, capable de moduler les aigus à des hauteurs vertigineuses, qui lui a valu le sobriquet de "rossignol". Pour d'autres, il évoque la Sape (Société des ambianceurs et personnes élégantes), mouvement de mode né dans les capitales jumelles des deux Congo, Brazzaville et Kinshasa, importé par les diasporas des villes européennes et diffusé jusqu'au Japon. D'autres encore l'associent à cette triste affaire de fraude à l'immigration, habilement montée en épingle dans un contexte de durcissement des politiques d'accueil des étrangers. Avec le recul, il semble bien que le chanteur congolais ait payé pour l'exemple.

\section{L'affaire}

2 Déclenchée en février 2003, ce qui allait devenir "l'affaire Papa Wemba" avait relégué au second plan le Sommet des chefs d'États africains dans les médias. Soupçonné d'avoir facilité, moyennant finance, l'entrée en France de plusieurs centaines de ressortissants de son pays la République démocratique du Congo (RDC), le chanteur passait trois mois et demi en prison préventive. "Cette pratique [faire entrer en Europe des personnes dans le cadre d'un groupe dont elles ne font pas partie] était courante, explique le chanteur. Non seulement dans le domaine de la musique, mais aussi dans les milieux religieux et sportifs, voire politiques. Tout le monde le faisait. Dans ma démarche, il ne s'agissait pas de gagner de l'argent. J'ai un métier avec lequel je gagne plutôt bien ma vie. Ce que j'ai aujourd'hui, je l'ai gagné à la sueur de mon front..."

3 Jugé pour "aide à l'entrée ou au séjour irrégulier, faux et usage de faux, et association de malfaiteurs", Papa Wemba a été condamné à 10000 d'amende et à 4 mois de prison avec sursis. Huit membres de son entourage étaient impliqués à ses côtés, dont sept écopèrent de faibles condamnations. À l'issue du procès, le chanteur et ses amis reçurent le soutien du chef de l'État congolais, Joseph Kabila, qui versa 30000 à l'État français. Certains disfonctionnements des administrations congolaises et françaises, qui 
étaient apparus au cours de l'instruction, n'ont pas été sanctionnés. Libre de ses mouvements après la sentence, Papa Wemba était toutefois fortement déstabilisé. "J'ai été malmené par la presse, surtout la presse française. Dans cette affaire, je pense avoir fait les frais de la politique", estime-t-il, amer. La principale conséquence de cette affaire fut l'arrêt brutal de sa carrière, qui pénalisait par ricochet l'économie de toute une communauté artistique contribuant aux activités du chanteur, répartie en Europe et à Kinshasa. "Mes problèmes judiciaires ont mis tout le clan à genoux, explique-t-il. Je suis pratiquement la seule personne qui apporte de l'argent dans mon clan. Tous les yeux de ses membres sont tournés vers moi."

4 Depuis de nombreuses années à Kinshasa, Papa Wemba a acquis le respect du petit peuple des démunis. "J'ai donné de l'espoir à un grand nombre de jeunes gens dans ce pays, poursuit-il. Hier on les appelait les 'enfants de la rue'. Dans mes chansons, je les ai appelés 'chégués'. Le nom leur est resté. Dans les années quatre-vingt, je jouais avec le groupe Viva la Musica tous les mercredis, samedis et dimanches au bar Vis-à-Vis, qui se trouve rond-point des Victoires à Kinshasa. Les concerts commençaient après minuit. Comme j'habite à dix ou quinze minute de là, les enfants de la rue venaient m'attendre devant chez moi. Quand je sortais, ils m'accompagnaient à pied jusqu'à l'entrée du bar. Ils restaient dehors. Je pénétrais dans le bar pour y faire mon travail jusque vers $4 \mathrm{~h} 30,5 \mathrm{~h}$ ou $6 \mathrm{~h}$ du matin. Quand je repartais, ils étaient là et me raccompagnaient jusque chez moi. Cette complicité n'a jamais disparu... J'ai aidé beaucoup de ces gamins des rues. J'ai pris certains d'entre eux par la main pour les emmener à l'école... Ici, les gens ont du mal à comprendre comment fonctionne notre société. Et c'est une des raisons pour lesquelles j'ai fait de la prison. Je n'ai jamais agi pour me remplir les poches. J'ai même payé de nombreux billets d'avions à des gens qu'il fallait extirper de la misère."

\section{Au-delà des apparences}

5 Les réalités de la vie des Congolais à Kinshasa sont parfois tout simplement inconcevables pour un Occidental. De ce point de vue, le film Benda Bilili ${ }^{1}$ (terme lingala qui signifie "au-delà des apparences"), unanimement applaudi en ouverture de la Quinzaine des réalisateurs au Festival de Cannes 2010, nous en dit long, sans discours superflu. Dès 2004, les réalisateurs, Renaud Barret et Florent de la Tullaye plongent au cœur de la capitale de la RDC, dans le chaos des laissés-pour-compte. Yeux et oreilles grands ouverts, dénués de tout préjugé, ils découvrent en immersion totale cette sorte d'inframonde qu'est celui d'un groupe de musiciens paraplégiques, jouant sur des instruments improbables : Staff Benda Bilili. Entourés d'enfants des rues qui partagent leur destin dans un soutien mutuel, ces musiciens sont mus par une volonté forcenée de s'en sortir. L'un des héros de cette édifiante aventure s'appelle Roger Landu. Ce garçon, âgé de 13 ans au début du film, tire des sons incroyablement musicaux d'un petit monocorde de son invention baptisé "satongé", fait d'une boîte de conserve et d'un manche de métal recourbé. Découvrant le talent du gamin, Ricky, le chef du groupe Staff Benda Bilili, décide de le former à la musique et de l'intégrer comme soliste. Leur optimisme n'a d'égal que la solidarité opiniâtre qu'ils mettent en œuvre pour tenter de s'extraire du chaos de Kinshasa. "La rue, c'est le monde très darwinien du Staff Benda Bilili. C'est là qu'ils travaillent, qu'ils dorment, qu'ils mangent, qu'ils élèvent leurs enfants, qu'ils créent leurs chansons et qu'ils répètent des nuits entières sous l'œil admiratif des enfants abandonnés dont ils sont devenus les 'papas' officieux", écrivent les cinéastes. Par leur entremise, 
Vincent Kénis du label Crammed Discs produit le premier disque et les tournées européennes du groupe.

\section{Le Village Molokaï}

6 Certes Papa Wemba ne vit pas dans la rue, mais il assume les mêmes responsabilités de rassembleur, dynamiseur, formateur, orienteur et employeur, vis-à-vis de toute la communauté artistique qui l'entoure et l'aide à gérer sa carrière. À Kinshasa dans le quartier populaire de Matonge, il a baptisé "Village Molokaii" le périmètre délimité par les rues perpendiculaires autour de la parcelle où s'installèrent ses parents au début des années cinquante. Cet espace d'habitation rudimentaire, fait d'une vaste pièce, sur laquelle donne une pièce plus petite, avec de grandes ouvertures sur une cour, où un coin est aménagé pour la cuisine, sert aujourd'hui de bureau, d'espace de rencontre et de répétition à l'ensemble des jeunes qui font partie du groupe kinois de Papa Wemba.

7 Ancien soldat de l'armée belge, son père devenu chasseur de gazelles, de buffles ou d'éléphants, s'opposait violemment à ce que Jules Shungu, aîné des fils de ses quatre femmes - et donc surnommé Papa - fasse de la musique. C'est sa mère, vendeuse de cacahuètes au marché mais aussi pleureuse dans les veillées mortuaires, qui fut son premier public et son premier professeur de chant. Enfant de chœur, sa voix s'élevait dans l'église Saint-Joseph, où il servait la messe. À la mort de son père en 1966, Papa Wemba devenait chef de famille. À 17 ans, sa passion pour la musique l'emportait. En cachette, il avait commencé à fabriquer des instruments de fortune, à animer des fêtes avec ses copains du quartier. Il faisait partie de ces "gembo" ("chauves-souris" en lingala) accrochés aux grilles extérieures des boîtes de nuit, où les stars de l'époque faisaient grandir la rumba congolaise, une musique de plus en plus originale et sophistiquée. Il adorait le rock et le rythm \& blues, au point que ses amis étudiants en Belgique le surnommaient "Jules Presley", avant qu'il ne démarre sa vraie vie de chanteur à 20 ans, en fondant le groupe Zaïko Langa Langa.

\section{Les orchestres écoles}

8 Aujourd'hui, plus d'une cinquantaine de jeunes chanteurs, musiciens, danseuses et danseurs, pour la plupart âgées de moins de 30 ans, apprennent leur métier auprès de Papa Wemba. Ce sont ses accompagnateurs et accompagnatrices sur les scènes du Congo et d'Afrique. Ils se forment sur le tas au sein de grands orchestres, avant de tenter leur chance avec d'autres compagnons ou en solo. En RDC, comme dans une bonne majorité de pays africains, il n'existe aucune école où apprendre les métiers de la scène. Les orchestres sont les seuls lieux d'encadrement où acquérir l'expérience de la musique pour devenir un bon professionnel. Viva la Musica, le groupe formé par Papa Wemba en 1977 et dont il est resté le leader, a vu défiler une pléiade de musiciens (Rigo Star, Sec Bidens, Awilo Longomba, Maïka Munan, etc.) et de chanteurs (Kester Emeneya, Dindo Yogo, Fafa de Molokaï, Stino Mubi, Luciana, Cele le Roi, Reddy Amisi, etc.). Il a également fait connaître la star Koffi Olomide en interprétant ses premières chansons, acquérant ainsi un autre sobriquet : "le formateur d'idoles".

9 À vrai dire, Papa Wemba n'a fait que perpétuer une pratique qui s'était instaurée dès la fin des années quarante avec la constitution d'orchestres de musique moderne autour 
des premières maisons de disques installées à Léopoldville (pas encore Kinshasa). Le système colonial belge ne laissant aux Africains aucune possibilité d'accéder à une formation spécialisée, les jeunes musiciens n'avaient d'autre choix de se former qu'au contact d'artistes plus expérimentés. Par exemple, c'est grâce au guitariste belge Bill Alexandre qu'a pu se répandre la pratique de la guitare électrique dans la musique congolaise. En 1953, après avoir mené une carrière fructueuse de jazzman en Europe, où il joua notamment aux côtés de Django Reinhardt, le musicien acceptait de prendre la direction du studio CEFA à Léopoldville. Il lui fallait constituer une équipe de musiciens congolais sous contrat avec le studio. Il initie alors de jeunes guitaristes, futurs grands talents comme Brazzos et Roitelet, qui révolutionneront le son des orchestres congolais de l'époque. "Il fallait d'abord que je leur apprenne à jouer en sol, en $\mathrm{fa}$, en la, en si, etc. Et très vite, ils devenaient des stars, parce que les autres guitaristes congolais s'étonnaient de les voir jouer dans tous ces tons et avec un médiator, ce qui était entièrement nouveau pour eux", se souvient Bill Alexandre ${ }^{2}$. On sait quel rôle primordial la guitare a joué dans l'évolution de la musique congolaise, notamment avec les géants de la six cordes Franco ${ }^{3}$ et Docteur Nico. Leurs groupes respectifs, OK Jazz et African Jazz, aux effectifs pléthoriques, servirent aussi à former musiciens et chanteurs. Et leur concurrence farouche se manifestait notamment dans l'habileté de chaque chef de groupe à subtiliser, dans le vivier de talents de l'autre, les éléments les plus prometteurs lorsqu'ils parvenaient à maturité.

\section{Nouvelles compositions}

10 La guitare demeure un instrument incontournable dans les chansons de Papa Wemba. "Pour composer, je fais toujours appel à des instrumentistes: le plus souvent un guitariste, car la guitare est l'instrument qui se marie le mieux au genre qu'est la rumba congolaise, confirme-t-il. Ma façon de travailler dans la chanson n'est jamais une initiative solitaire. J'ai toujours avec moi des collaborateurs. C'est une part de la force de ma musique. En effet, je ne suis pas musicien, je ne sais jouer d'aucun instrument, mais seulement de ma voix." Le retour de Papa Wemba, c'est donc aussi celui de tout son collectif artistique. Il se décline sous deux formes, deux tendances reflétant l'évolution de la carrière du chanteur.

11 Notre Père Rumba s'accorde à l'exubérance de Kinshasa. Il reçoit les contributions d'une vingtaine de chanteuses et chanteurs, quatre animateurs, onze guitaristes, cinq bassistes et un contrebassiste, six percussionnistes et trois batteurs, deux claviers et un pianiste, sans parler de la section de cuivres. C'est un disque tourbillon dont le tempo torride réveille la passion de la danse. Un disque qui n'a pas peur de conjuguer l'évocation de l'amour avec le pathos langoureux de l'âme populaire. Un disque très africain aussi, où s'égraine la litanie des noms de personnes honorées par le chanteur, le plus souvent selon leur qualité de généreux donateurs. Un disque dans lequel, et pour la première fois depuis longtemps, Papa Wemba utilise dans une chanson les rythmes farouchement prenants et la langue de ses ancêtres Tetela, tribu faisant partie du grand groupe des Anamongo, dont il avait été intronisé "Grand notable" par les Chefs coutumiers à l'occasion de ses 50 ans.

12 Notre Père World s'inscrit dans le prolongement des trois albums publiés par le label Real World de Peter Gabriel, dont le sublime Émotion, paru en 1995. Il fait appel à un orchestre restreint, dont se dégagent les sonorités limpides d'une guitare acoustique ou 
le son d'un piano. Les arrangements sobres forment comme un écrin valorisant la texture si particulière de la voix de Papa Wemba. Il en émane une mélancolie poignante sur le texte de Simple rêve, tourné à la manière de ce qu'il y a de meilleur dans la chanson française. Elle transporte un mystère quasi ésotérique sur les vers espagnols de Tonanda de Luna Llena, complainte du Vénézuélien Simon Diaz popularisée par Caetano Veloso, pour laquelle Papa Wemba a eu le coup de foudre. "Voilà près de trois ans que je travaille sur cet album. J'écoute, j'essaye d'imaginer. Je me projette très loin”, explique le chanteur. Alors que Ma Rosa, dédiée à sa femme, privilégie le velouté crooner, d'autres morceaux, comme Azanga, chanté avec le griot guinéen Sekouba Bambino, vous transporte sur les rythmes de la forêt. Quant à Nako Kité, cette création du jeune ingénieur $\mathrm{du}$ son kinois Madou, fait résonner le génie du funk US façon "blackspoitation" 70's avec cette touche urbaine incomparable que seule la ville de Kinshasa est capable de produire. La richesse de l'album réserve encore beaucoup de belles surprises. "Je crois que ce qui va faire la force de cet album, c'est l'ensemble des différentes informations qu'il contient. Celui qui l'écoutera en intégralité ne va pas s'ennuyer, prédit Papa Wemba. Je crois qu'il me ressemble."

\section{BIBLIOGRAPHIE}

Papa Wemba, Notre Père Rumba (Sina Performance / Cantos, 2010)

Papa Wemba, Notre Père World (Sina Performance / Cantos, 2010)

Staff Benda Bilili, Très Très Fort (Crammed Discs, 2009)

\section{NOTES}

1. Sorti en salle le 8 septembre

2. Gary Stewart, Rumba on the River, ed. Verso, p. 42

3. Cf. $H \& M n^{\circ} 1266$ et 1267

\section{RÉSUMÉS}

Après sept ans de traversée du désert, Papa Wemba renoue avec le disque, la scène et ses publics. Notre Père est le titre commun aux deux albums jumeaux parus en juillet et octobre 2010. L'un, sous-titré Rumba, comble l'appétit de ses fans congolais et africains. L'autre, sous-titré World, ravira toute oreille sensible à ses prodigieuses qualités de chanteur. Son retour en grâce dans les réseaux professionnels est marqué par sa participation aux deux grands salons marchés des 
musiques du monde, Babel Med Music, fin mars à Marseille, et le Womex, fin octobre à Copenhague. À 61 ans, convaincu qu'il a encore beaucoup à donner musicalement, Papa Wemba éclaire certains aspects de sa démarche artistique. 